\title{
UM AMBIENTE DE SIMULAÇÃO DE INVENTÁRIO PARA UM CENTRO DE DISTRIBUIÇÃO DE PEÇAS
}

\section{A SIMULATE ENVIROMENT TO INVENTORY OF A PART DISTRIBUTION CENTER}

\author{
Heráclito Lopes Jaguaribe Pontes ${ }^{1}$; Arthur José Vieira Porto ${ }^{2}$ \\ ${ }^{1}$ Escola de Engenharia de São Carlos da USP - EESC/USP - São Carlos/SP - Brasil \\ hjpontes@sc.usp.br \\ ${ }^{2}$ Escola de Engenharia de São Carlos da USP - EESC/USP - São Carlos/SP - Brasil \\ ajvporto@sc.usp.br
}

\begin{abstract}
Resumo
Atualmente as empresas tentam alcançar altos volumes de produção e distribuição usando o mínimo de inventário durante toda a cadeia logística. Para diminuir o inventário total e distribuir os produtos em curto período de tempo, muitas empresas têm centro de distribuição em sua rede de distribuição. Para se alcançar um melhor desempenho no gerenciamento do inventário de um centro de distribuição é importante ter uma ferramenta dedicada a realizações de simulações de possíveis cenários. O objetivo deste trabalho foi desenvolver um ambiente de simulação de inventário para um Centro de Distribuição de Peças (CDP) específico. O ambiente de simulação realiza projeções visando obter como resultados a previsão de demanda, a política de reposição das peças, o percentual de atendimento dos pedidos, os meses de inventário, a quantidade de scrap e a quantidade de peças para devolução. Para o desenvolvimento do ambiente de simulação utilizou-se métodos de modelagem e linguagem de programação orientada a objeto. $O$ ambiente de simulação foi submetido a experimentos com três cenários diferentes e os resultados confirmaram a qualidade do sistema de simulação proposto.
\end{abstract}

Palavras-chave: centro de distribuição de peças, inventário, simulação.

\section{Introdução}

A tomada de decisões faz parte do dia-a-dia das empresas. $\mathrm{O}$ alto grau de competitividade no meio empresarial exige a capacidade de tomar decisões rápidas e precisas. A qualidade da tomada de decisão tem relação direta com os dados disponíveis para o tomador de decisão e com sua habilidade em extrair desses dados informações relevantes. No gerenciamento de um inventário diversas decisões são necessárias, com isso os dados e informações devem estar disponíveis e confiáveis. 
A eficácia do gerenciamento do inventário tem grande influência na lucratividade do Centro de Distribuição de Peças (CDP) e na empresa como um todo. A capacidade de gerenciar o inventário determina diretamente os níveis de estoques necessários para atingir os níveis de serviços desejados. Para alcançar o melhor desempenho no gerenciamento do inventário é importante que o CDP tenha uma ferramenta que permita realizar simulações dos principais indicadores gerenciais do inventário. A partir destas simulações, os responsáveis pelo inventário terão condições de tomar decisões com maior segurança e rapidez.

O CDP em estudo possui um sistema computacional de controle do inventário. Este sistema é rígido e seus dados são mantidos concisos e seguros, e serve para controlar todas as transações do inventário. Entretanto, esse sistema não permite que se realizem simulações que possibilitem aos responsáveis pelo inventário realizarem estudos a partir da variação dos dados de entrada para posterior acompanhamento do comportamento dos indicadores do inventário.

A aplicação do ambiente de simulação de inventário no CDP tem como objetivo fazer estudos de simulação para se obter como resultados a previsao de demanda, a política de reposição das peças, o percentual de atendimento dos pedidos completos, o valor do inventário, a quantidade de Scrap (refugo) e quantidade de peça para devolução. O ambiente de simulação possibilita aos responsáveis pelo inventário avaliar diferentes políticas gerenciais sem impactar ou interromper os processos em andamento no dia-a-dia do CDP.

A empresa utilizada como base para o desenvolvimento do ambiente de simulação é uma multinacional localizada no estado de São Paulo. Em seu CDP no Brasil ocorrem atualmente cerca de 50.000 Calls (atendimentos) por mês ou cerca de 2.000 Calls diários e para atender esta demanda o inventário do CDP é composto por aproximadamente 100.000 peças.

\section{Revisão Bibliográfica}

Para o desenvolvimento do ambiente de simulação de inventário para o CDP proposto neste trabalho, foi necessário o entendimento de conceitos relacionados a centro de distribuição de peças, inventário e simulação.

\subsection{Centro Distribuição de Peças (CDP)}

O Centro de Distribuição de Peças (CDP) é definido como um espaço físico responsável pela armazenagem de uma variedade de peças de um fornecedor ou da própria fábrica e responsável também pela liberação das peças para seus clientes (MULCAHY, 1994, p.12). 
De acordo com Frazelle (2002, p.3), CDP é o local onde se armazena peças de um fabricante ou de diversos fabricantes por um determinado período de tempo, para serem consolidados e enviados aos seus consumidores em comum.

Para Gurgel (1996, p.98), as expedições das fábricas tendem a evoluir para CDP, expedindo peças provenientes de várias fábricas de uma corporação, produtos comprados de terceiros e mesmo peças importadas. Os CDP devem ficar em áreas de fácil acesso e sua localização e seu tamanho dependerão da área atingida pelo CDP, da população concentrada na área e do poder aquisitivo.

\subsubsection{Principais Funções de um CDP na Cadeia Logística}

De acordo com Tompkins et al. (1996, p.391), existem diversos tipos de armazéns dentre eles o CDP. De acordo com a localização destes armazéns no contexto logístico, estes podem ter diferentes funções. Em geral, as funções dos CDP são:

- Permitir o equilíbrio entre a quantidade produzida e a demanda;

- Diminuir a distância de transporte para permitir uma resposta aos clientes em menor tempo;

- Permitir a consolidação de produtos de vários fornecedores para consumidores comuns, ou seja, tornar os produtos mais próximos à manufatura e ao consumo.

Segundo Farah Junior (2002, p.45), os CDP são instrumentos que viabilizam de forma competitiva o fluxo de peças vindas dos fabricantes, até os seus diversos graus de capilaridade distributiva. O CDP deve ser um meio de minimização de custos, melhoria no uso dos recursos e apoio ao processo de venda e pós-venda.

De acordo com Alvarenga e Novaes (2000, p.121), sob o ponto de vista logístico, o CDP pode ter diversas funções, dependendo dos objetivos da empresa. Suas principais funções são as seguintes:

- Armazenagem propriamente dita: esta é a função mais comum. Existem casos em que há necessidade ou conveniência de estocar os produtos por um tempo relativamente grande e outros casos em que a armazenagem é apenas de passagem. Há também situações mistas.

- Consolidação: é o processo de reunir cargas parciais provenientes de origens diversas para formar carregamentos maiores. Isso ocorre porque é mais barato transportar lotações completas e maiores para médias e longas distâncias, do que enviar a carga em lotes pequenos, diretamente a partir das várias origens.

- Fracionamento: é o processo inverso da consolidação, em que carregamentos maiores são desmembrados em pequenos lotes para serem encaminhados a destinos diferentes. 


\subsubsection{Vantagens da Utilização de um CDP}

Diversas vantagens são identificadas na literatura quanto à adoção do CDP no sistema logístico. Essas vantagens obtidas pela armazenagem, centralização de estoque podem beneficiar todos os elos da cadeia: fornecedor, empresa e consumidor.

Segundo Farah Junior (2002, p.46), algumas vantagens de um CDP são: cumprimento do tempo de entrega, precisão no atendimento, qualidade do produto entregue, suporte no pós-venda, redução do custo de transporte, liberação de espaço nas fábricas e nos clientes, redução de mão-deobra nos clientes para o recebimento e conferência de mercadorias e diminuição de falta de produtos nos clientes.

Bowersox e Closs (2001, p.326) identificam também duas vantagens na adoção do CDP no sistema logístico: a capacidade de agregar valor ao produto (postergação) e os diferentes tipos de operações que podem ser realizadas no mesmo (Consolidação de cargas, Break Bulk, Cross-Dock e Formação de Estoque).

\subsection{Inventário}

O inventário consiste em uma reserva de matérias-primas, materiais em processo e produtos acabados ou peças que se encontram em inúmeros lugares dentro de uma empresa e em canais logísticos. São freqüentemente encontrados em CDP, em equipamentos de transporte e em pontos de vendas.

Segundo Cox III e Blackstone Junior (2002, p.57), inventário são aqueles estoques usados para suportar produção (matéria-prima e itens em processo), suportar atividades gerais (manutenção, reparos e suprimento de operações) e suportar serviço aos clientes (produto acabado, partes e peças).

\subsubsection{Gerenciamento de Inventário}

Conforme Krajewski e Ritzman (2004, p.295), o gerenciamento de inventário é uma preocupação importante para os gerentes em todos os tipos de empresa. Por essa razão, os gerentes acompanham de perto os inventários para mantê-los em níveis aceitáveis. O desafio consiste não em diminuir os estoques de forma excessiva para reduzir custos ou ainda ter muito estoque disponível a fim de satisfazer todas as demandas, mas em possuir a quantidade certa para alcançar as prioridades competitivas da empresa do modo mais eficiente. A função do gerenciamento de inventário consiste em compatibilizar os custos e as pressões conflitantes que exigem estoques reduzidos e níveis de serviços elevados (KRAJEWSKI e RITZMAN, 2004, p.295). 
De acordo com Gaither e Frazier (2002, p.269) há muitas razões pelas quais é importante ter altos níveis de inventário, mas também há razões pelas quais a manutenção desses inventários elevados é considerada imprudente. Os principais motivos para manter altos níveis de inventário são: serviço ao cliente, custo de pedido, custo de falta de estoque, custos de aquisição, custos de transporte. Os principais motivos para a não manutenção de altos níveis de inventário são: custos de armazenagem e manuseio, custos de oportunidade, custos para coordenar a produção, custos de redução do retorno sobre o investimento, imposto, seguros e perdas.

Segundo Brewer et al. (2001, p.196), as organizações têm que responder três questões importantes no gerenciamento de inventário que são: que itens devem ser estocados, quando deve ser realizado um novo pedido do item e qual tamanho deve ser o pedido. Complementa Robeson e Copacino (1994), que gerenciamento de inventário é composto de três componentes chaves: controle do inventário, previsão de demanda e reposição de estoque. $\mathrm{O}$ foco do controle do inventário é mais o presente e o passado, enquanto a previsão de demanda e a reposição do estoque tratam mais do futuro.

\subsection{Simulação}

Simulação computacional ou simulação é o processo de projetar um modelo lógico matemático de um sistema real e fazer experimentos deste sistema no computador (PRITSKER, 1986, p.6).

Conforme Shannon (1998, p.7), simulação consiste no processo de desenvolver um modelo de um sistema real e realizar experimentos com este modelo com o propósito de entender o comportamento do sistema e/ou as evoluções das várias estratégias para a operação do sistema.

Para Harrel e Tumay (1997, p.39), simulação é uma atividade por meio da qual se pode tirar conclusões sobre o comportamento de um dado sistema pelo estudo do comportamento do seu modelo correspondente no qual as relações de causas e efeitos são as mesmas ou simulares do sistema real.

\subsubsection{Quando usar Simulação}

Segundo Freitas Filho (2001, p.4), a simulação permite ao analista realizar estudos sobre os correspondentes sistemas para responder questões do tipo "O que aconteceria se". O principal apelo ao uso desta ferramenta, é que tais questões podem ser respondidas sem que os sistemas sob investigação sofram qualquer perturbação, uma vez que os estudos são realizados no computador. A simulação permite que tais estudos sejam realizados sobre sistemas que ainda não existem, levando ao desenvolvimento de projetos eficientes antes que qualquer mudança física tenha sido iniciada. 
Bertrand e Fransoo (2002, p.255) apontam que apesar da qualidade científica dos resultados da simulação ser menor que o caso de análise matemática, sua relevância cientifica é alta. A variedade de modelos científicos na qual a simulação é capaz de trabalhar é maior que uma análise matemática. Eles apontam que a simulação é utilizada em casos onde os modelos ou problemas são muitos complexos para uma análise matemática formal.

\subsubsection{Vantagens e Desvantagens da Simulação}

As vantagens da simulação são citadas por Banks (1998, p.10), Banks et al. (1996, p.4), Kelton et al. (1998, p.7), Carson II (2004, p.3) e Centeno e Carrillo (2001, p.18) são relacionadas abaixo:

- Modelos mais realistas: maior liberdade na construção do modelo;

- Processo de modelagem evolutivo;

- Uma vez criado, um modelo pode ser utilizado inúmeras vezes para avaliar projetos e políticas propostas;

- Os modelos de simulação podem ser quase tão detalhados quanto os sistemas reais, novas políticas e procedimentos operacionais, regras de decisão, fluxos de informação, podem ser avaliados sem que o sistema real seja perturbado;

- Perguntas do tipo "e se?" (“what if?”): em diversos casos, em lugar de buscar uma solução, o objetivo resume-se em tornar mais claras as possíveis conseqüências de um conjunto de decisões;

- Aplicação a problemas mal-estruturados;

- Grande flexibilidade: a simulação aplica-se aos mais variados problemas;

- Aquisição de visão sistêmica.

Os mesmos autores citam também algumas desvantagens da simulação:

- A modelagem e a análise da simulação podem ser dispendiosas em termos de recursos financeiros e de tempo;

- A construção de modelos requer treinamento especial;

- A programação de um modelo de simulação pode ser tornar altamente dispendiosa e desgastante se os recursos computacionais não forem apropriados;

- Os resultados da simulação são, muitas vezes, de difícil interpretação;

- Pode ser usada inapropriadamente, por exemplo, quando uma solução analítica é factível. 


\section{Ambiente de Simulação de Inventário para Centro de Distribuição de Peças}

O ambiente de simulação desenvolvido neste trabalho complementa o sistema gerencial de inventário do CDP em estudo realizando simulações futuras dos indicadores gerenciais sem agredir a integridade dos dados contidos no sistema gerencial já existente. A partir destas simulações, os responsáveis pelo inventário têm condições de tomar decisões com maior segurança e mais rapidamente. $\mathrm{O}$ ambiente possibilita aos mesmos avaliar diferentes políticas gerenciais sem impactar ou interromper os processos em andamento no dia-a-dia do CDP.

O desenvolvimento do ambiente de simulação foi baseado na metodologia de simulação de Freitas Filho (2001, p.13) que é dividida nos seguintes passos: formulação e análise do problema; planejamento do projeto; formulação do modelo conceitual; coleta de macro-informações e dados; tradução do modelo; verificação e validação; projeto experimental; experimentação; interpretação e análise estatística dos resultados; comparação de sistemas e identificação da melhor solução; documentação e apresentação dos resultados e implementação.

A modelagem do simulador foi realizada empregando a UML (Unified Modeling Language) através do software Rational Rose e a implementação foi realizada na linguagem de programação $\mathrm{C}++$ (Borland $\mathrm{C}++$ Builder). Para entrada e saída de dados utilizou-se a própria interface do simulador e planilha do Microsoft Excel.

\subsection{Dados de Entrada do Ambiente de Simulação}

O ambiente de simulação possui entradas por interface e por planilha do Microsoft Excel. As entradas por interface são divididas em dois grupos: interface geral (variáveis de entrada utilizadas por todas as peças) e interface fonte (variáveis de entrada que diferem de acordo com o local de origem da peça, ou seja, nacional ou importada).

A entrada por arquivo (planilha do Microsoft Excel) é realizada devido à necessidade de utilização no ambiente de simulação de variáveis de entrada com valores individuais para cada peça, ou seja, cada peça tem características individuais que entram no programa pelo arquivo peça.

Os dados de entrada por interface são determinados pelo operador do inventário e os dados de entrada por planilha do Microsoft Excel são fornecidos diretamente do sistema de gerenciamento de inventário do CDP.

Os principais dados de entrada por interface geral são: mês atual; horizonte de simulação; dias úteis dos meses de simulação; taxa do dólar; previsão da variação do mercado; previsão de promoção de vendas; prazo máximo de permanência no CDP; freqüência para devolução das peças e valor disponível para devolução de peças. 
Os principais dados de entrada por interfaces fonte são: estoque em trânsito; tempo de consolidação de pedidos; política de estoque máximo; política de estoque mínimo; lead time; e coeficiente de suavização.

Os principais dados de entrada por planilha do Microsoft Excel são: código da peça; código do inventário; lote mínimo de compra; múltiplo de embalagem; peso unitário; custo unitário; preço de venda; backorder (ordens em atraso); família da peça; estoque físico das peças; previsão de demanda para o primeiro mês de simulação; desvio para o primeiro mês de simulação; a demanada histórica de calls (atendimentos) dos últimos 36 meses; e quantidade de peças por calls dos últimos 36 meses.

\subsection{A Lógica de Funcionamento do Ambiente de Simulação}

O ambiente de simulação executa seqüencialmente e de maneira repetitiva um conjunto de instruções. Na medida em que as instruções são executadas, os valores das variáveis são alterados, uma vez que se modificam as condições que influenciam o comportamento do ambiente.

O ambiente de simulação inicia quando o operador do inventário solicita acesso ao sistema. O sistema verifica se ele tem autorização, em caso positivo é liberado o acesso ao sistema. Se a autorização for negativa, o operador solicita login e senha para o administrador do sistema. Após entrar no ambiente de simulação, o operador vai ao menu inventário e cria os arquivos de entrada de dados: fonte nacional, fonte importada e geral. No mesmo menu inventário, o operador pode visualizar o arquivo peça (planilha do Microsoft Excel). Ainda no mesmo menu, o operador seleciona a opção simular e após abrir todos os arquivos de entrada (fonte nacional e importada, geral e arquivo peça), inicia-se a simulação.

A simulação inicia com a criação de uma lista de peças para inserção dos dados de cada peça. Em seguida, o código de cada peça é formado. De acordo com o código formado a peça é classificada. Os dados de entrada de cada peça são inseridos na lista de peças de acordo com a classificação da peça. Após a sua classificação, o sistema inicia o cálculo da projeção da demanda real da peça.

Depois de classificar as peças, projetar suas demandas reais para todo o horizonte de simulação e inserir todos os dados na lista de peças, inicia-se a segunda fase da simulação. Na segunda fase da simulação, o sistema realiza o cálculo da Reposição de Peças, o cálculo do Percentual de Atendimento de Pedidos, o cálculo dos Meses de Inventário, o cálculo do Scrap, o cálculo da Devolução de Peças e o cálculo da Previsão de Demanda para cada peça do inventário. O horizonte de simulação determina a quantidade de vezes que vão ser executadas estas operações. Após a simulação gera-se um arquivo de resultados com os dados de cada peça na extensão do horizonte de simulação. 


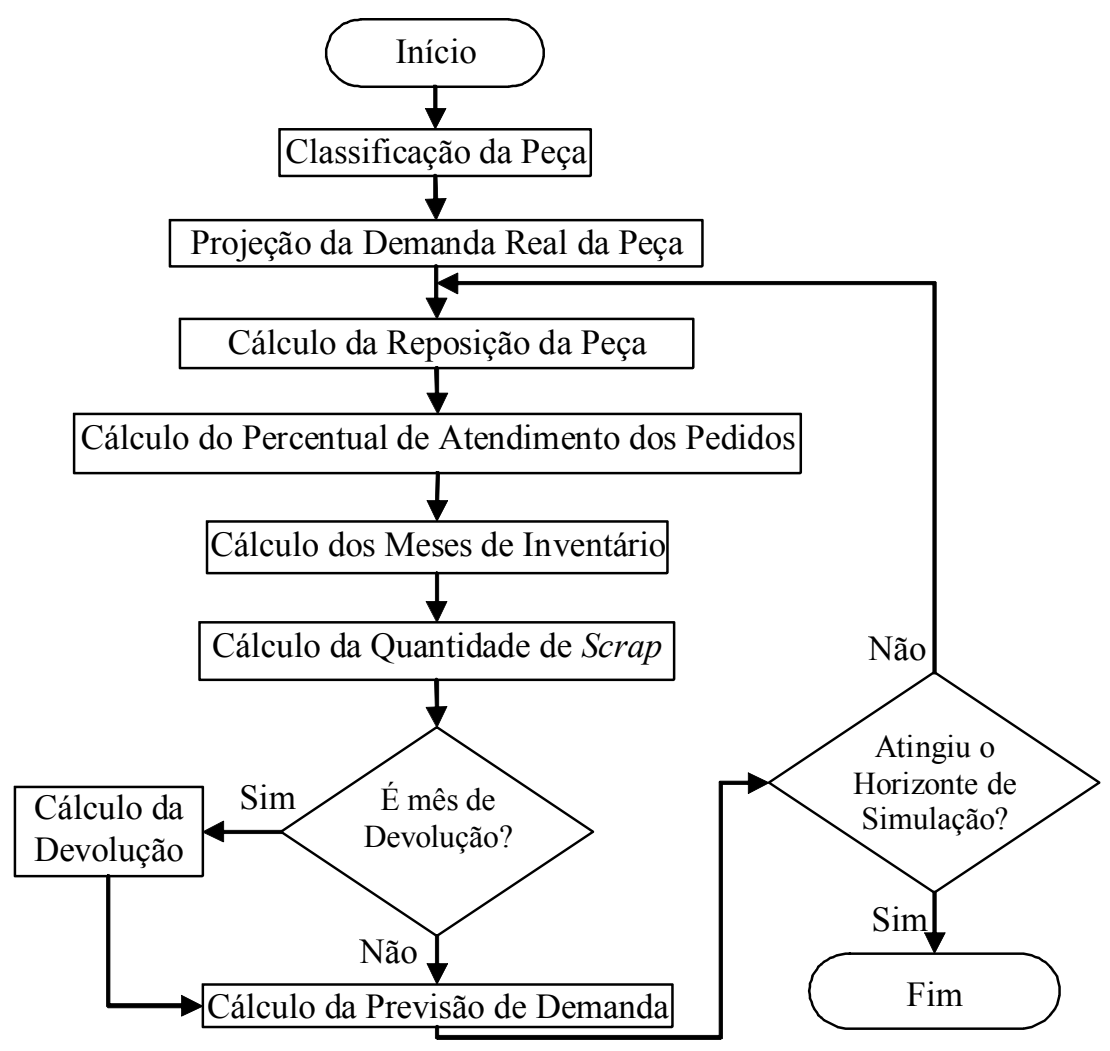

\subsubsection{Classificação das Peças}

O código da peça para o gerenciamento do inventário é formado a partir da entrada dos dados pela planilha do Microsoft Excel. Após a formação do código inicia-se a classificação. A classificação da peça consiste nas seguintes etapas:

- Primeiramente, verifica-se se a peça é desimpactada ou impactada. A peça é classificada como desimpactada quando o número de Calls (atendimentos) nos últimos doze meses é menor que três, ou seja, a peça não tem mais uma demanda significativa, com isso será descontinuada;

- O segundo passo da classificação é verificar qual a fonte da peça, nacional ou importada. As peças nacionais são divididas em dois tipos: peças produzidas na própria fábrica da empresa e as peças compradas de fornecedores nacionais;

- O último passo da classificação da peça é a verificação de qual classe a peça pertence. As classes são divididas em A, B, C de acordo com sua representatividade nas vendas e Normal e Proteção (proteção são peças que ficam estocadas no inventário devido ao lançamento de novas máquinas no mercado). 


\subsubsection{Projeção da Demanda Real}

Para cada mês do horizonte de simulação é necessário ter uma demanda, como não é possível obter a demanda real de meses futuros, optou-se por calcular a projeção da demanda real baseando-se em métodos quantitativos baseados em séries temporais.

O cálculo da projeção da demanda real consiste em, a partir do histórico da demanda de 36 meses, projetar a demanda de acordo com o horizonte de simulação. Essa projeção é realizada considerando diversos fatores como tendência, sazonalidade, promoção de venda, crescimento ou diminuição de mercado, de acordo com a Figura 2.

Figura 2 - Fluxograma da Projeção da Demanda Real

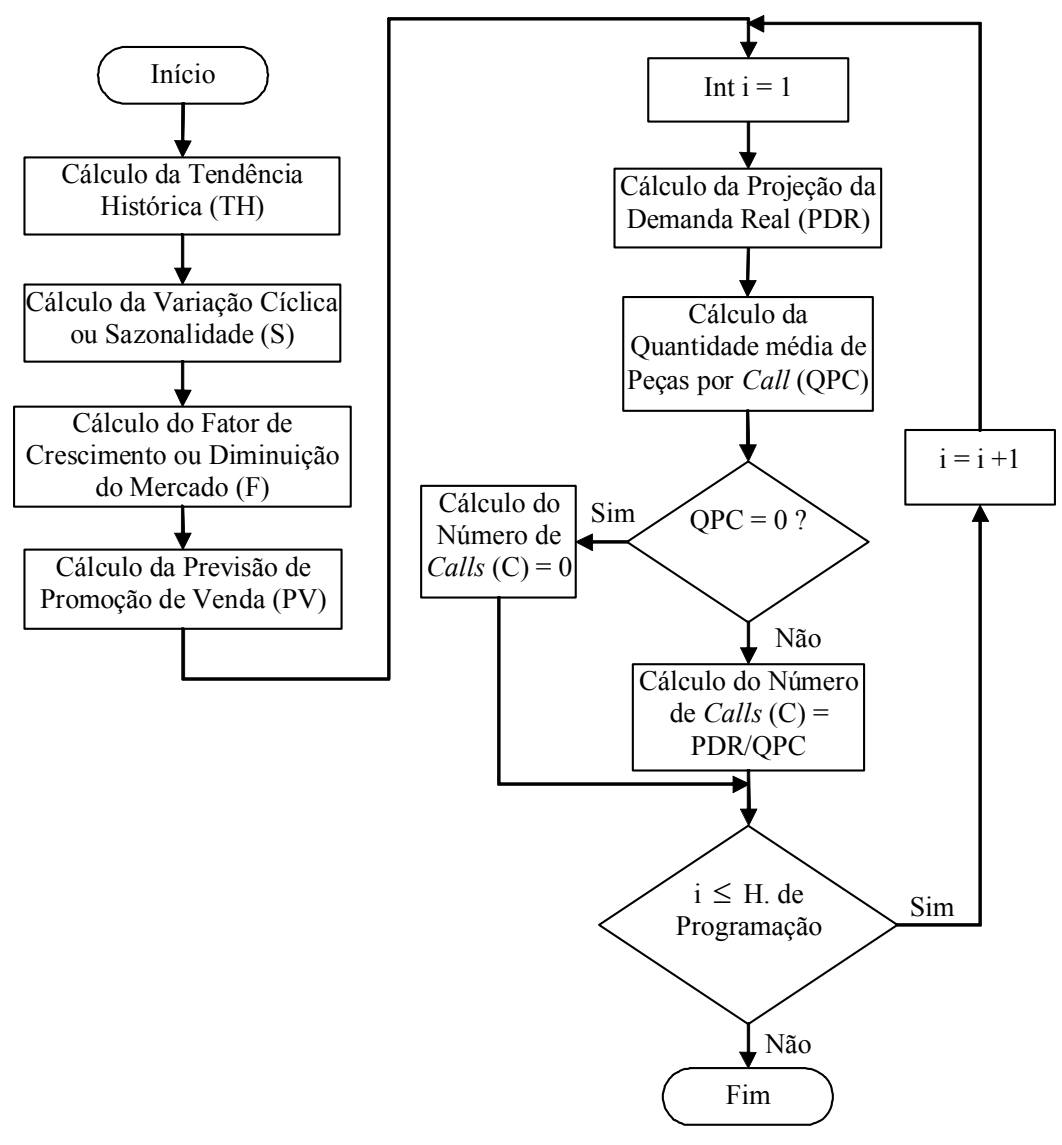

Inicialmente calcula-se a tendência histórica (TH) que é composta pela média móvel mensal da demanda, o acréscimo ou decréscimo mensal da demanda e o número de meses do horizonte de simulação. A segunda etapa é o cálculo da variação cíclica ou sazonalidade (S). A sazonalidade é obtida pelo cálculo do desvio médio real da demanda. A terceira etapa é o cálculo do fator de crescimento ou diminuição mensal do mercado (F). No início da simulação o operador do simulador determina o valor de previsão de crescimento ou diminuição do mercado e também determina em quais meses vai haver o crescimento ou diminuição do mercado. 
A quarta etapa é cálculo da promoção de venda (PV). No início da simulação o operador do simulador determina o valor da variação percentual das vendas sobre a demanda de cada mês do ano, ou seja, determina o percentual de previsão de promoção de vendas de cada mês do ano.

A projeção da demanda real (PDR) para cada mês do horizonte de simulação é obtida a partir de todas as etapas anteriores. Após o cálculo de PDR, realiza-se o cálculo da quantidade média de peças por Calls (QPC) para cada mês do horizonte de simulação. O QPC é obtido pela média dos QPC do mesmo período da demanda histórica de 36 meses. A partir do cálculo de PDR e QPC obtém-se o número de Calls $(\mathrm{C})$ para cada mês do horizonte de simulação.

\subsubsection{Reposição das Peças}

A reposição da peça do CDP tem como objetivo determinar quanto e quando repor a peça. As peças desimpactadas não têm uma política de reposição de peças devido ao fato de não ter uma demanda significativa. Em alguns casos pode haver a necessidade de reposição de estoque somente para atender as ordens em atraso (backorders).

A política de reposição de peça adotada para as peças impactadas utiliza recursos dos dois principais modelos para demanda independente que são: o modelo de revisão periódica e o modelo de revisão contínua

A política de reposição de cada peça impactada sofre variações de acordo com a classificação da peça, mas basicamente o funcionamento do sistema é o seguinte:

- Quando a posição de estoque de cada peça atinge um nível mínimo (estoque mínimo), uma quantidade de peça é solicitada para repor o estoque para um nível máximo (estoque máximo). O cálculo do estoque mínimo, estoque máximo e da quantidade de peça varia de acordo com a classificação da peça;

- Para não ocorrer falta de estoque do momento em que se faz o pedido até o recebimento da peça utiliza-se um estoque de segurança. Cada peça do inventário tem um valor de estoque de segurança diferente.

\subsubsection{Nível de Serviço ao Cliente}

Para o gerenciamento do nível de serviço ao cliente utiliza-se o indicador percentual de atendimento de pedidos (PAP) que é o percentual de pedidos atendidos em sua íntegra para cada mês do horizonte de simulação.

Para obtenção do PAP de cada peça calcula-se o número de Calls $(\mathrm{C})$ no mês em que está ocorrendo a simulação. Se C for maior que zero, calcula-se a possibilidade de atendimento de 
pedido (POP). O valor de POP é o inteiro resultante da divisão do estoque disponível total (EDT) pelo número de peças por Call (QPC) do mês em que está ocorrendo a simulação.

Se o valor de POP for igual a zero, significa dizer que não há estoque disponível da peça para atender os Calls e o PAP também será igual a zero. Se o valor de POP for maior que zero, calcula-se o PAP que é o resultado da divisão da POP por C. Multiplica-se o resultado por 100 para transformar o número em percentual. Se o valor de PAP for maior que 100, então, limita-se PAP a 100, ou seja, PAP $=100 \%$ (maior nível de serviço possível).

Figura 3 - Fluxograma do Percentual de Atendimentos de Pedidos

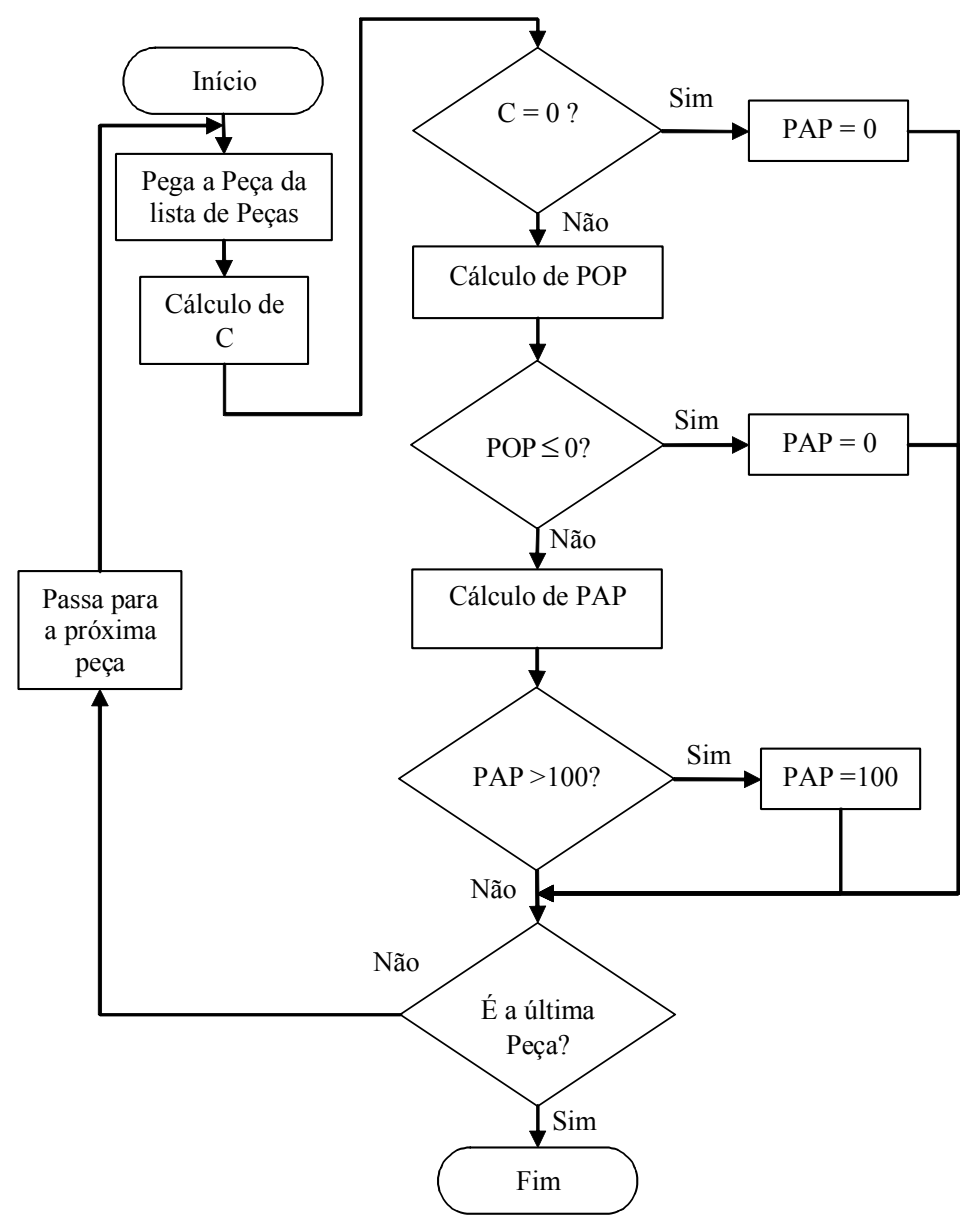

\subsubsection{Meses de Inventário}

Para o gerenciamento do valor investido no inventário utiliza-se o indicador meses de inventário (MI) que é a quantidade de meses que o estoque de cada peça duraria, sujeito às vendas futuras, sem que haja reposição do estoque da peça.

Quando o valor de MI é pequeno, corre-se o risco de faltar peça para o atendimento ao cliente e conseqüentemente reduzir o nível de satisfação do cliente. Quando o valor de MI é grande, corre-se o risco de ter estoques obsoletos e aumento dos custos de manutenção de estoque. A determinação do valor de MI varia de peça para peça dependendo da sua importância para o CDP. 
Para obtenção do MI de cada peça calcula-se o estoque disponível total (EDT). Se EDT for maior que zero, calcula-se o estoque disponível total em reais (EDTR).

Após calcular o EDTR, calcula-se a média das vendas dos últimos quatro meses (MV). Se EDTR ou MV for igual a zero, MI também será igual a zero. Se EDTR e MV forem maiores que zero, o MI é obtido pela divisão de EDTR por MV.

Figura 4 - Fluxograma dos Meses de Inventário

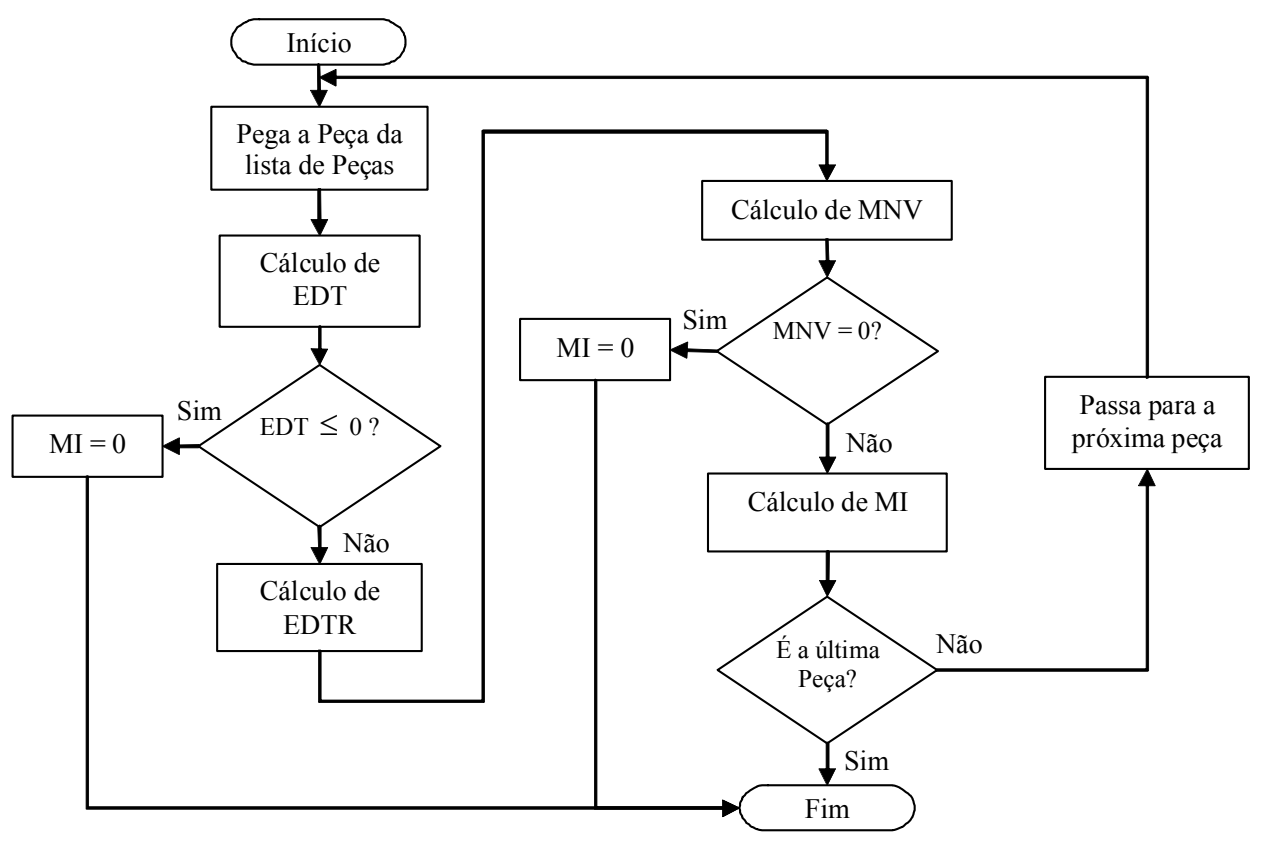

\subsubsection{Scrap}

As peças são consideradas Scrap quando não podem ser usadas para o seu propósito original, mas têm um certo valor para o inventário. As etapas para o cálculo das peças Scrap são as seguintes:

- Inicialmente cria-se uma lista virtual denominada lista de peças Scrap para inserir todas as peças consideradas candidatas a Scrap. A próxima etapa é verificar se a peça é Scrap. Calcula-se a demanda histórica (DH) dos últimos trinta e seis meses da peça. Comparase o DH com o índice de excesso sem demanda (IESD). O IESD é determinado na entrada de dados geral pelo operador do ambiente de simulação no início da simulação. Se DH for menor que IESD, denomina-se a peça como candidata a Scrap e adiciona a mesma na lista de peças Scrap;

- Se a lista de Scrap já tiver alguma peça, então as peças são ordenadas segundo uma regra que pode ser: por maior peso, por menor peso, por maior custo unitário, por menor custo unitário. A determinação de qual regra vai ser utilizada é realizada no início da simulação na interface de dados geral pelo operador do inventário; 
- Na próxima etapa, calcula-se o valor do estoque de cada peça (VEP) candidata a Scrap. Após calcula-se a cota mensal de Scrap (CMS). A CMS é determinada pelo operador do ambiente de simulação no início da simulação na interface de dados geral;

- Se o VEP for menor ou igual à CMS, desconta-se o valor do VEP da CMS. No indicador de quantidade de Scrap da peça (QSP) no mês atual de simulação é colocado o valor do VEP e no estoque físico coloca-se zero. Se após esse procedimento ainda houver saldo na CMS, aplica-se o mesmo procedimento na próxima peça. Se não houver mais saldo na CMS, zeram-se os marcadores de candidato a Scrap.

Figura 5 - Fluxograma Scrap

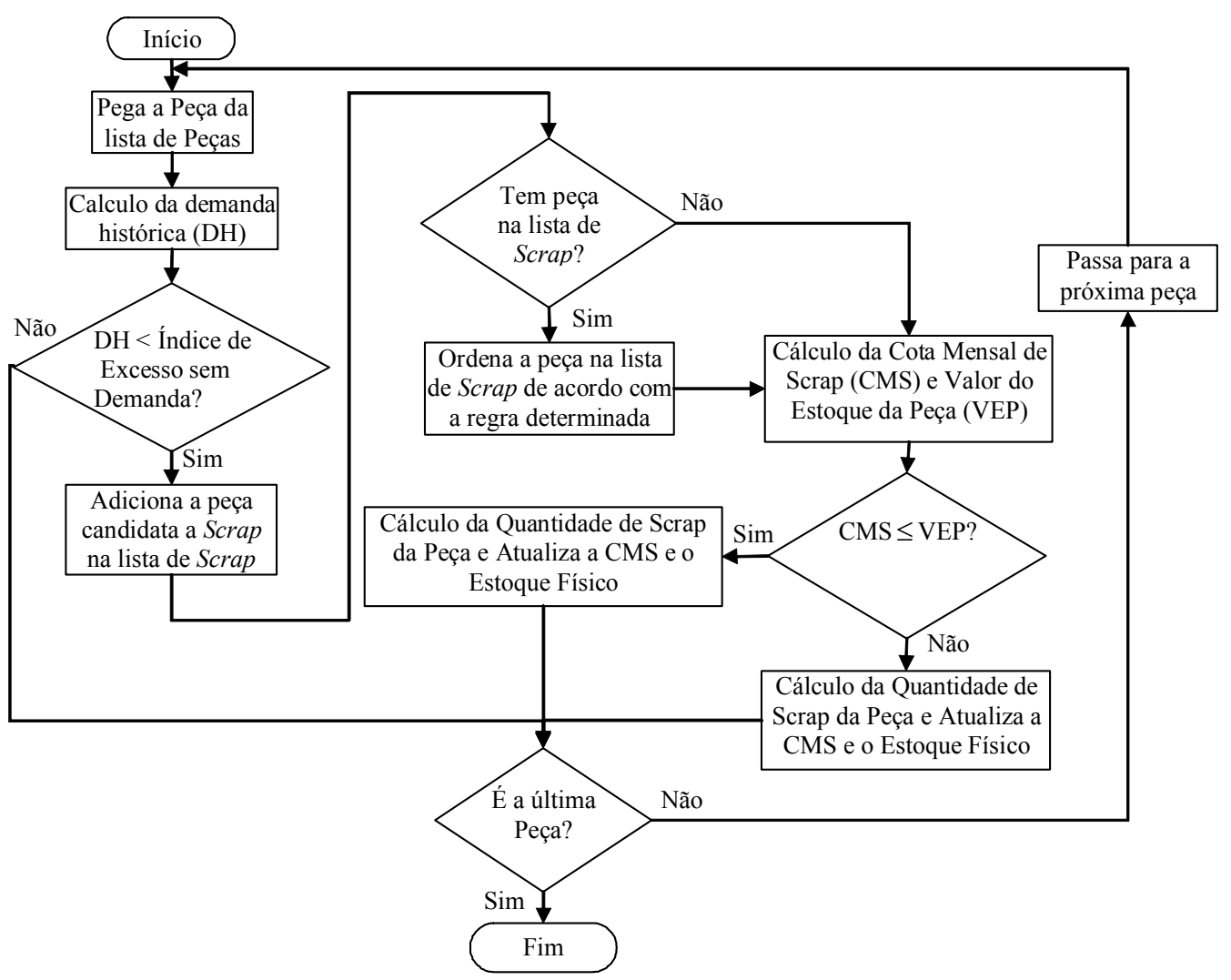

\subsubsection{Devolução de Peças}

A devolução da peça é realizada quando a mesma não apresenta uma demanda histórica condizente com seu estoque. A devolução ocorre quando o estoque da peça é maior do que a demanda histórica dos últimos 36 meses. No início da simulação o operador do ambiente determina a freqüência para devolução das peças. A freqüência pode ser mensal, bimestral, trimestral, quadrimestral, semestral ou anual.

Na operação de devolução de peças, o CDP já desembolsou uma quantia monetária (valor da peça e impostos) para ter aquela peça em seu estoque, com isso é necessário calcular a quantidade 
de devolução e o valor em reais dessa quantidade de devolução. O cálculo da quantidade para devolução e do valor de devolução é realizado da seguinte forma:

- Nos meses determinados como de devolução, calcula-se o valor total disponível para devolução em reais (VTD) e pega a peça da lista de peças. Em seguida, verifica se o estoque físico da peça (DPF) é maior que zero. Caso o DPF seja maior que zero, calculase a demanda histórica dos últimos 36 meses da peça (DH) e compara com o DPF. Se a quantidade do estoque físico da peça for maior que a demanda histórica, calcula-se a quantidade de peça para devolução (QPD) e o valor de devolução da peça em reais (VDP);

- Na próxima etapa, compara-se o valor de devolução da peça em reais (VDP) com o valor disponível para devolução em reais (VTD). A partir desta comparação atualiza-se a quantidade de peça para devolução, o valor disponível para devolução e o estoque físico da peça. Essa etapa é realizada para todas as peças classificadas como devolução até a finalização do valor disponível para devolução.

Figura 6 - Fluxograma da Devolução de Peças

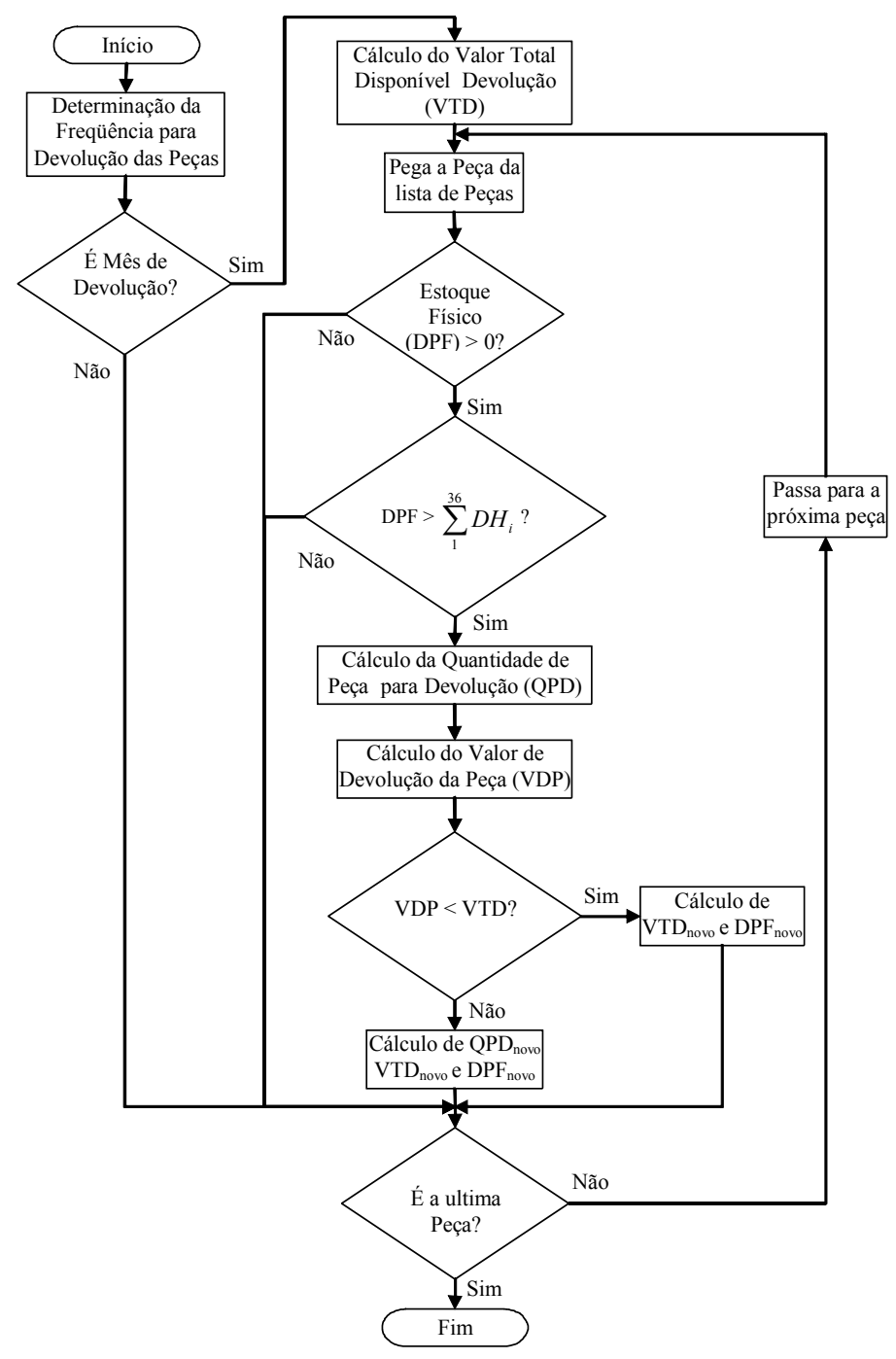




\subsubsection{Previsão de Demanda}

A previsão de demanda é realizada em todo final do mês de simulação com o objetivo de prever o mês seguinte. A previsão de demanda é realizada baseada no método de suavização exponencial simples. Para realizar o cálculo da previsão de demanda mensal do próximo mês de simulação utiliza-se a demanda real atual, a previsão de demanda atual e o coeficiente de suavização.

A demanda real e a previsão de demanda no primeiro mês de simulação são fornecidas pela planilha do Microsoft Excel. Após o primeiro ciclo de simulação utiliza-se a demanda real projetada e utiliza-se a previsão calculada no primeiro ciclo de simulação e assim por diante em todos os meses de simulação até completar o horizonte de simulação. O coeficiente de suavização é determinado pelo operador do simulador para cada fonte de peça (nacional ou importada) no início da simulação e seu valor deve estar entre zero e um

Figura 7 - Fluxograma da Previsão de Demanda

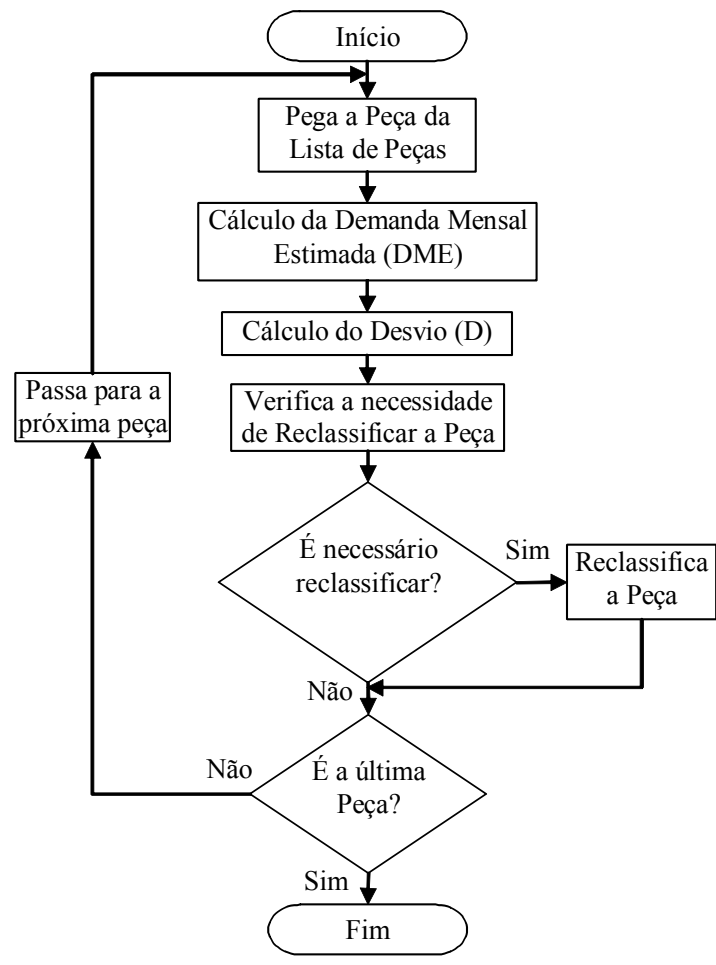

Além da previsão de demanda, realiza-se o monitoramento da previsão de demanda e reclassifica-se a peça quando necessário. O monitoramento é realizado com o cálculo do desvio entre a demanda real e a demanda prevista. O desvio é também calculado no final de cada mês. Para o calculo do desvio do primeiro mês de simulação utiliza-se o desvio fornecido pela planilha do Microsoft Excel. Após o primeiro ciclo de simulação utiliza-se sempre o desvio anterior e assim por diante em todos os meses de simulação até completar o horizonte de simulação. Além do desvio 
anterior, o desvio é composto pelo erro ponderado, desvio real, desvio previsto e o coeficiente de suavização.

A última etapa da previsão de demanda é verificar a necessidade de reclassificar a peça e definir o momento necessário para realizar a reclassificação. No simulador há dois tipos de reclassificação possíveis que são: a peça pode ser impactada ou desimpactada; a peça pode ser classificada como A, B ou C.

\subsection{Dados de Saída do Ambiente de Simulação}

O ambiente de simulação oferece a possibilidade da saída de dados em planilha do Microsoft Excel ou via interface do próprio ambiente. Os dados de saída são:

- Código do Inventário: é o código utilizado para o gerenciamento do inventário;

- Código da Peça: é o código da peça utilizado para localização da peça no inventário;

- Família: é a família à qual a peça pertence;

- Número de Calls: é a quantidade de Calls de cada mês do horizonte de simulação;

- Quantidade de Peças por Calls: é a quantidade de peças por Calls de cada mês do horizonte de simulação;

- Backorders: são os Calls de cada peça que não foram atendidos durante cada mês do horizonte de simulação;

- Reposição da Peça: é quantidade a ser reposta de cada peça durante o horizonte de simulação;

- Recebimento da Peça: é quantidade de recebimento de cada peça durante o horizonte de simulação;

- Meses de Inventário: é a quantidade de meses de inventário de cada peça durante o horizonte de simulação;

- Percentual de Atendimento de Pedidos: é o percentual de atendimentos de pedidos de cada peça durante o horizonte de simulação;

- Estoque das Peças: é o estoque das peças durante o horizonte de simulação;

- Devolução das Peças: é a quantidade devolvida aos fornecedores das peças pertencentes durante o horizonte de simulação;

- Scrap: é a quantidade de peças Scrap em cada mês do horizonte de simulação;

- Previsão de Demanda: é a previsão de demanda estimada da peça em cada mês do horizonte de simulação;

- Desvio: é o desvio acumulado entre a demanda real e a demanda prevista da peça em cada mês do horizonte de simulação. 


\subsection{Experimentação no Ambiente de Simulação}

Para verificar o bom funcionamento do simulador foram desenvolvidos três cenários com diferentes dados de entrada e horizontes de simulação. A quantidade de peças simuladas foi à mesma para os três cenários (1.000 peças). Os três cenários simulados apresentaram resultados condizentes com o sistema real. O horizonte de simulação não influenciou nos resultados finais, ou seja, para o simulador não importa a quantidade de meses que se está simulando, mas sim os parâmetros de entrada.

Com os resultados deste trabalho comprovou-se que o ambiente de simulação condiz com a realidade, assim o mesmo esta pronto para fazer projeções futuras e o resultado estará condizente desde que os dados de entradas correspondam ao histórico, e avaliações de mercado atuais e projetadas correspondam realmente aos acontecimentos futuros.

\section{Considerações Finais}

Este trabalho apresentou um ambiente de simulação de inventário para um CDP específico que permite fazer previsões de demanda, da política de reposição das peças, do percentual de atendimento dos pedidos, do valor do inventário, da quantidade de Scrap, da quantidade de peças para devolução, sempre com a preocupação de interagir de forma flexível e mantendo a integridade dos dados e variáveis originais.

A realização deste trabalho evidenciou algumas vantagens do uso do ambiente de simulação de inventário para o CDP em estudo, que foram:

- A possibilidade de avaliar diferentes políticas gerenciais do inventário do CDP sem impactar ou interromper os processos em andamento no mundo real, o que poderia produzir custos adicionais significativos;

- A possibilidade de comprimir o tempo, permitindo aos responsáveis pelo inventário conhecer em pouco tempo, as conseqüências de longo prazo de suas ações;

- A possibilidade de melhor entender o funcionamento dos processos gerenciais do inventário do CDP individualmente, assim como suas influências sobre o sistema como um todo;

- A utilização de interfaces simples e amigáveis que permitem a manipulação dos dados de entrada e saída e a realização da simulação facilmente e eficazmente;

- A observação da importância de cada peça do inventário, assim como a sinalização de quando a peça deve ser descontinuada ou devolvida ao fornecedor;

- A possibilidade de melhor avaliar o desempenho do gerenciamento do inventário; 
As principais dificuldades encontradas durante a realização do trabalho foram:

- A coleta de dados ocorreu com dificuldade devido o CDP em estudo ter um política de privacidade de dados;

- Realização da projeção da demanda futura das peças.

\begin{abstract}
Nowadays the companies try to get high volumes of production and distribution using the minimum of inventory during all the supply chain. To reduce the total inventory and to distribute the products in short period of time, many companies have a distribution center for distribution. To get better performance in the management of the inventory of a distribution center it is important to have a dedicated tool to the accomplishments of simulation of possible scenes. The objective of this work was to develop an environment of simulation of inventory for a Parts Distribution Center (PDC) specific. The simulation environment performs projections in order to get demand forecasts, the parts replenishment policy, the attendance percentage, the inventory months, scrap quantity and quantity part to devolution. To develop the simulation environment were used tools like modeling methods and programming object-oriented language. The simulator was submitted to experiments with three different sceneries and the results confirmed the quality of the simulation system proposed.
\end{abstract}

Key-words: part distribution center, inventory, simulation.

\title{
Referências
}

ALVARENGA, A. C; NOVAES, A. G. N. Logística aplicada: suprimento e distribuição física. 3.ed. São Paulo: Edgar Blucher Ltda, 2000.

BANKS, J. Handbook of simulation: principles, methodology, advances, applications, and practice. New York: John Wiley \& Sons, 1998.

BANKS, J.; CARLSON, J. S.; NELSON, B. L. Discrete-event system simulation. 2.ed. New Jersey: Prentice-Hall, 1996.

BERTRAND, J. W. M; FRANSOO, J. C. Modeling and Simulation: Operations management research methodologies using quantitative modeling. International Journal of Operations \& Production Management, v.22, n.2, p.241-264, 2002.

cross

BOWERSOX, D. J.; CLOSS, D. J. Logística empresarial: o processo de integração da cadeia de suprimento. São Paulo: Atlas, 2001.

BREWER, A.M., et al. Handbook of logistics and supply-chain management. Kidlington: Elsevier Science Ltd, 2001.

CARSON II, J, S. Introduction to modeling and simulation. Proceedings of the 2004 Winter Simulation Conference, p.01-08, 2004.

CENTENO, M.A.; CARRILLO, M. Challenges of introducing simulation as a decision making tool. Proceedings of the 2001 Winter Simulation Conference, p.17-21, 2001.

COX III, J.F.; BLACKSTONE JUNIOR, J.H. American Production Inventory Control Society Dictionary. 9.ed. Falls Church: American Production and Inventory Control Society, 2002.

FARAH JUNIOR, M. Os desafios da logística e os centros de distribuição física. Revista FAE BUSINESS, n.2, p.4446, 2002. 
FRAZELLE, E. World-class warehousing and material handling. New York: McGraw-Hill, 2002.

FREITAS FILHO, P.J. Introdução à modelagem e simulação de sistemas: com aplicação em Arena. Florianopolis: Visual Books, 2001.

GAITHER, N.; FRAIZER, G. Administração da Produção e Operações. 8.ed. São Paulo: Pioneira, 2002.

GURGEL, F. A. Administração dos fluxos de materiais e de produtos. São Paulo: Atlas, 1996.

HARREL, C.; TUMAY, K. Simulation made easy. IIE Solutions, p.39-41, July, 1997.

KELTON, W. D. et al. Simulation with arena. New York: WCB/McGraw Hill, 1998.

KRAJEWSKI, L.J; RITZMAN, L. P. Administração da Produção e Operações. São Paulo: Prentice Hall, 2004.

MULCAHY, D. E. Warehouse distribution \& operations handbook. New York: McGraw-Hill, 1994.

PRITSKER, A. A.B. Introduction to Simulation and SLAM II. New York : John Wiley \& Sons, 1986.

ROBESON, J.F.; COPACINO, W.C. The logistics handbook. New York: The Free Press, 1994.

SHANNON, R. E. Introduction to the art and science of simulation. In: Proceedings of the 1998 Winter Simulation Conference. p.07-14, 1998.

TOMPKINS, J. A. et al. Facilities planning. 2.ed. New York: John Wiley \& Sons, 1996.

\section{Dados dos autores:}

Heráclito Lopes Jaguaribe Pontes

Escola de Engenharia de São Carlos da Universidade de São Paulo - EESC/USP

Departamento de Engenharia Mecânica

Doutorando em Engenharia Mecânica pela EESC/USP

Avenida Trabalhador Sãocarlense, 400 - Centro, São Carlos- SP - Brasil - 13566-590

(16) - 3373-9432

hjpontes@sc.usp.br

Arthur José Vieira Porto

Escola de Engenharia de São Carlos da Universidade de São Paulo - EESC/USP

Departamento de Engenharia Mecânica

Professor Titular

Avenida Trabalhador Sãocarlense, 400 - Centro, São Carlos- SP - Brasil - 13566-590

(16) - 3373-9432

ajvporto@sc.usp.br 